\title{
Reconfigurable Generation of High-Order Ultra-Wideband Waveforms Using Edge Detection
}

\author{
Avi Zadok, Member, OSA, Xiaoxia Wu, Student Member, IEEE, OSA, \\ Jacob Sendowski, Student Member, IEEE, OSA, Amnon Yariv, Life Fellow, IEEE, OSA, and \\ Alan E. Willner, Fellow, IEEE, OSA
}

\begin{abstract}
The generation of ultra-wideband (UWB), high-frequency waveforms based on nonlinear propagation of pulses in optical fibers is reported. Self-phase modulation and subsequent optical filtering are used to implement all-optical edge detectors, which generate two temporally-narrowed replicas of each input pulse. The shapes of the narrowed pulses are subtracted from that of the original one in a balanced differential detector, providing a UWB waveform. The use of multiple replicas and nonlinear propagation allows for the generation of higher-order pulse shapes, beyond those of a Gaussian monocycle or doublet. The output pulse shape is reconfigurable through adjusting the input power and detuning the optical filters. The central radio-frequency of the generated waveforms is as high as $34 \mathrm{GHz}$, with a fractional bandwidth of $70 \%$.
\end{abstract}

Index Terms-Microwave photonics, nonlinear fiber-optics, optical signal processing, ultra-wideband pulse generation.

\section{INTRODUCTION}

$\mathbf{U}$ LTRA-WIDEBAND (UWB) radio is a transmission technology that is based on short pulses, whose spectral width is on the order of several GHz. UWB signals are free of sinewave carriers, and their duty cycle and power spectral density are low. These characteristics provide UWB radio with unique advantages: improved immunity to multi-path fading, increased ranging resolution, large tolerance to interfering legacy systems, enhanced ability for penetrating obstacles, and low processing power. UWB technology is considered attractive for a myriad of applications, including high-speed internet access, sensor networks, high accuracy localization, precision navigation, covert communication links, ground-penetrating radar, and throughthe-wall imaging [1].

Manuscript received November 01, 2009; revised January 13, 2010, February 01, 2010; accepted February 02, 2010. Date of publication February 17, 2010; date of current version July 26, 2010. This work was supported in part by the National Science Foundation (NSF) ERC CIAN. The work of A. Zadok was supported in part by a post-doctoral fellowship from the Center for the Physics of Information (CPI), California Institute of Technology.

A. Zadok was with the California Institute of Technology, Pasadena, CA 91125 USA. He is now with the School of Engineering, Bar-Ilan University, Ramat-Gan, 52900 Israel (e-mail: zadoka@eng.biu.ac.il).

$\mathrm{X}$. $\mathrm{Wu}$ and $\mathrm{A}$. E. Willner are with the Department of Electrical Engineering-Systems, University of Southern California, Los Angeles, CA 90089-2565 USA (e-mail: xiaoxia@usc.edu; willner@usc.edu).

J. Sendowski is with the Department of Electrical Engineering, California Institute of Technology, Pasadena, CA 91125 USA (e-mail: jacobsen@ caltech. edu).

A. Yariv is with the Department of Electrical Engineering and the Department of Applied Physics, California Institute of Technology, Pasadena, CA 91125 USA (e-mail: ayariv@caltech.edu).

Color versions of one or more of the figures in this paper are available online at http://ieeexplore.ieee.org.

Digital Object Identifier 10.1109/JLT.2010.2042790
Of the various potential UWB radio applications, much attention has turned to wireless personal area networks, which address short-range, ad-hoc, and high-rate connectivity among portable electronic devices. UWB radio is well suited to take the place of cables in such networks, due to its multi-path and interference tolerance, low power, and high efficiency. Research efforts in this area have intensified since 2002 when the United States Federal Communication Commission (FCC) allocated the frequency range of 3.6-10.1 GHz for unlicensed, UWB indoor wireless communication [2]. Interest is not limited to indoor wireless communication only: the FCC report relates to imaging systems and vehicular radar systems as well [2]. The latter, in particular, are specified for a high central frequency of $24 \mathrm{GHz}$ or higher [2]. The electronic generation of complex UWB waveforms at such high frequencies is increasingly challenging.

The FCC standard imposes several limitations on the transmitted signals. First, the power spectral density must comply with complicated spectral masks [2]. In addition, the signal power is also severely restricted, limiting the range of UWB wireless transmission, for example, to only $10-15 \mathrm{~m}$. In many cases, a UWB radio-based system would have to complement wireless transmission by other distribution means, and optical fibers would be the medium of choice for high-frequency signals. With imminent radio-over-fiber integration, the generation of the UWB pulses by photonic methods becomes attractive. Microwave-photonic generation techniques can enable flexible tuning of the pulse shapes. Electronic filtering, on the other hand, does not provide equivalent pulse reconfiguration. Driven by the promise of integration and flexibility, much research effort has been dedicated to photonic generation of UWB waveforms in recent years [3]-[13].

One category of UWB generation techniques relied on the conversion of phase to intensity modulation [3]-[5]. This method is simple to implement, however it offers few degrees of freedom for pulse shaping and minimal reconfiguration. Waveforms generated using this method are restricted to a Gaussian mono-cycle or a Gaussian doublet shape. Higher-order pulse shapes were generated based on microwave-photonic tapped delay line filters, with both positive and negative coefficients [6]-[8]. Pulse generation based on four-coefficients filters had been demonstrated [8], however each additional coefficient required an extra laser source. Another interesting approach is based on relaxation oscillations in directly-modulated or externally-injected distributed feedback lasers [9], [10]. High-order waveforms were recently generated using these methods, however their frequency range is restricted by relaxation oscillations to the order of $10 \mathrm{GHz}$. The most elaborate waveform 
tailoring was provided by optical spectrum shaping and subsequent frequency-to-time mapping [11]-[13]. These techniques required mode-locked laser sources, and either bulky free-space optics [12] or highly complex fiber gratings with limited tuning [13]. Simple optical platforms for UWB generation, which are scalable to higher-order waveforms, reconfigurable and utilize standard off-the-shelf components, are yet to be proposed. Such methods, if developed, may considerably enhance UWB radio-over-fiber integration.

Nonlinear propagation effects in optical fibers are powerful tools for optical signal processing. Examples include pulse compression and regeneration using self-phase modulation (SPM) [14], slow light-based delay lines [15], and microwave photonic filters based on stimulated Brillouin scattering [16], [17]. However, nonlinear propagation in optical fibers was seldom used in UWB pulse generation research. In [18], Li and coauthors used cross-gain modulation in an optical parametric amplifier to generate monocycle and doublet pulse shapes. Velanas et al. [19] used a cross-phase-modulation (XPM) based technique to obtain monocycle shapes. Both schemes required two input laser sources.

In this work, we use nonlinear propagation of a pulse train from a single laser source for the generation of high-order UWB waveforms. All-optical edge detectors of the original pulses intensity are used to generate two temporally-narrowed replicas of the input pulse train. The edge detection relies on the timevarying chirp introduced by SPM, and judiciously tuned optical filters. SPM accumulates through propagation along sections of fiber, which can also serve for the distribution of pulses from a network terminal to a remote antenna element. The shapes of the narrowed replicas are subtracted from that of the original pulse train in a broadband, balanced differential detector. The resulting waveforms are highly reconfigurable through adjustments of the input power and tuning of the optical filters. High-order UWB waveforms having a center frequency of 34 $\mathrm{GHz}$ and a fractional bandwidth of $70 \%$ are generated. The analysis and measurements provide a significant extension to previous works utilizing nonlinear propagation in fibers [18]-[21], which were restricted to monocycle and doublet pulse shapes of lower central frequencies. The results provide a first demonstration, to the best of our knowledge, of high-order UWB waveforms generation with a single laser source and off-the-shelf components.

\section{Self-Phase Modulation Based Edge Detection}

Consider the optical field $E_{\text {in }}(t)$ of an input train of superGaussian pulses:

$$
E_{\text {in }}(t)=\sum_{n} \sqrt{P_{\text {in }}} \exp \left[-\left[\frac{\left|t-n T_{0}\right|}{2 \tau_{0}}\right]^{m}\right] \exp \left(j \omega_{0} t\right)
$$

with a peak power level $P_{\text {in }}$, central optical frequency $\omega_{0}$, width parameter $\tau_{0}$ and pulse separation $T_{0}$. The parameter $m$ determines the exact shape of the input pulses. In propagating along a highly nonlinear fiber (HNLF) of length $L$ and negligible dispersion, the optical field undergoes SPM:

$$
\begin{aligned}
E_{\mathrm{HNLF}}(t) & =E_{\mathrm{in}}(t) \exp [j \varphi(t)] \\
& =E_{\mathrm{in}}(t) \exp \left[j \gamma L\left|E_{\mathrm{in}}(t)\right|^{2}\right],
\end{aligned}
$$
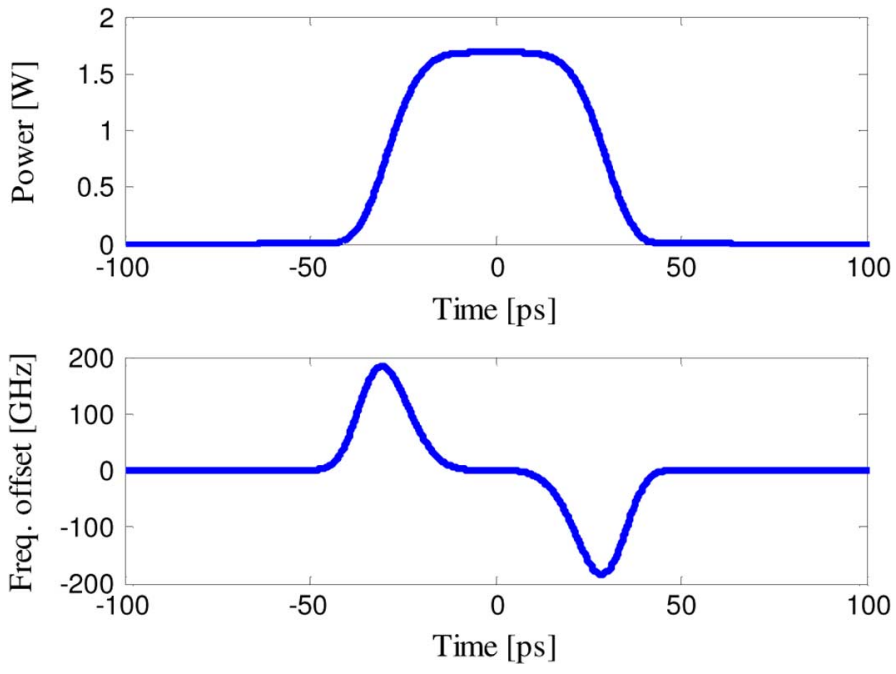

Fig. 1. Top-instantaneous power of an input super-Gaussian pulse: $m=$ $5, \tau_{0}=27 \mathrm{ps}, P_{\text {in }}=1.7 \mathrm{~W}$. Bottom-simulated SPM-induced instantaneous frequency shift $\Delta f_{\text {pulse }}(t): L=1 \mathrm{~km}, \gamma=11.3[\mathrm{~W} \cdot \mathrm{km}]^{-1}$.

where $\gamma[\mathrm{W} \cdot \mathrm{km}]^{-1}$ is the nonlinear coefficient of the fiber. The nonlinearly induced phase modulation $\varphi(t)$ represents an effective temporally-varying shift of the optical frequency (chirp):

$$
\Delta f_{\text {pulse }}(t)=\frac{1}{2 \pi} \frac{\mathrm{d} \varphi(t)}{\mathrm{d} t}=\frac{\gamma L}{2 \pi} \frac{\mathrm{d}\left|E_{\mathrm{in}}(t)\right|^{2}}{\mathrm{~d} t} .
$$

Fig. 1 shows the instantaneous power $\left|E_{\text {in }}(t)\right|^{2}$ of a single input super-Gaussian pulse with $m=5$ (top panel), and the corresponding $\Delta f_{\text {pulse }}(t)$ for a $1 \mathrm{~km}$-long HNLF with $\gamma=11.3$ $[\mathrm{W} \cdot \mathrm{km}]^{-1}$ (bottom panel). As seen in the figure, the leading (trailing) edge of the pulse is associated with a positive (negative) frequency shift.

Edge detection of $E_{\mathrm{HNLF}}(t)$ is implemented by an optical bandpass filter (BPF) of spectral width $2 \delta$, detuned from $\omega_{0}$ by a frequency offset $\Delta \omega>\delta>0$. The BPF would block most of the waveform, except for a segment of sufficient SPM: $2 \pi \cdot \Delta f_{\text {pulse }}(t)>\Delta \omega-\delta$. As seen in (3), this segment corresponds to the leading edge of the pulse. The BPF therefore represents an all-optical intensity edge detector. The details of the narrowed replica of the pulse at the BPF output are determined by its spectral width and detuning as well as the input power $P_{\text {in }}$. The endpoints of the filtered waveform $t_{1,2}$ are approximately given by: $\mathrm{d}\left|E_{\text {in }}\left(t_{1,2}\right)\right|^{2} / \mathrm{d} t=(\Delta \omega-\delta) / \gamma L$. The trailing edge can be filtered in a similar manner, with $\Delta \omega<0,|\Delta \omega|>\delta>$ 0 .

Fig. 2 shows the instantaneous power $\left|E_{ \pm}(t)\right|^{2}$ at the output of $75 \mathrm{GHz}$-wide BPFs, whose central frequencies are detuned from $\omega_{0}$ by $\Delta \omega_{ \pm} / 2 \pi= \pm 135 \mathrm{GHz}$, respectively. As expected, the filtered waveforms emphasize the pulse edges, and both are narrower than the original input pulse. Unlike previous schemes for all-optical intensity differentiation, which were based on XPM in fiber [19] or cross-gain modulation in semiconductor optical amplifiers [22], the proposed edge detection technique does not require an additional probe input. The shape of the two narrowed replicas can be subtracted from that of the original pulse to generate an UWB waveform, as described next. 


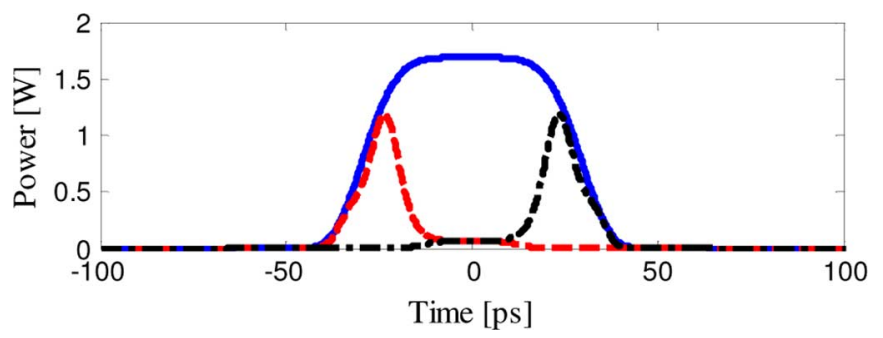

Fig. 2. Solid-instantaneous power of an input super-Gaussian pulse (same as top panel of Fig. 1). Dashed-simulated instantaneous pulse power following propagation in a HNLF and filtering by a $75 \mathrm{GHz}$-wide BPF, detuned from $\omega_{0}$ by $\Delta \omega_{+} / 2 \pi=135 \mathrm{GHz}$. HNLF parameters are the same as those in the bottom panel of Fig. 1. Dashed-dotted-same as dashed curve, with the BPF detuned from $\omega_{0}$ by $\Delta \omega_{-} / 2 \pi=-135 \mathrm{GHz}$

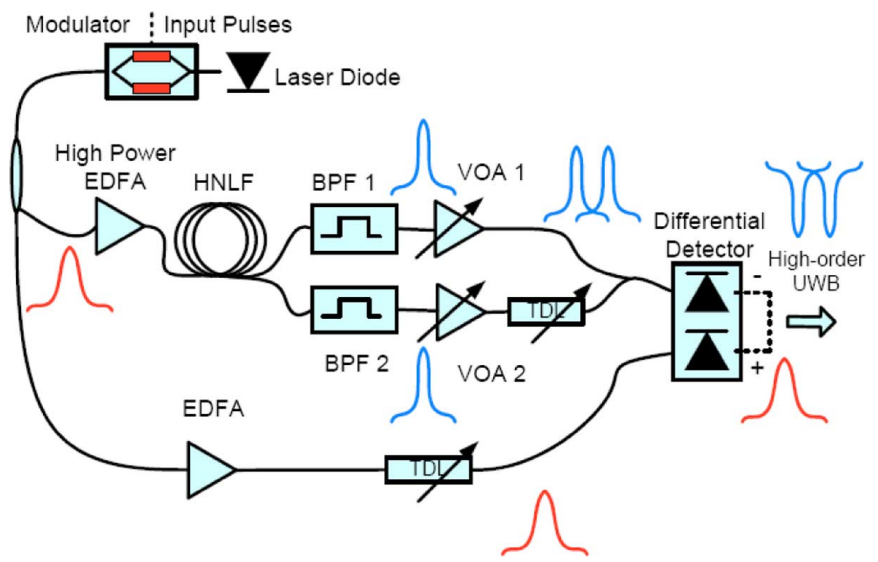

Fig. 3. Schematic diagram of the UWB pulse generation scheme. EDFA: erbium-doped fiber amplifier; HNLF: highly nonlinear fiber; BPF: bandpass filter; VOA: variable optical attenuator; TDL: tunable delay line.

\section{ULTRA-WIDEBAND WAVEFORM GENERATION}

Fig. 3 shows a schematic drawing of a setup for UWB waveform generation, based on all-optical edge detection. The input super-Gaussian pulse train is split in two branches. The upper branch includes a high-power erbium-doped fiber amplifier (EDFA) and an HNLF section. At the HNLF output, the spectrally broadened pulses are split into two paths once again, and the light in each path is filtered by an individually tunable BPF. BPF 1 is tuned to detect the pulse leading edge as discussed above, whereas BPF 2 is adjusted as a trailing edge detector. The power level of each of the two pulse train replicas is individually adjusted by a variable optical attenuator (VOA). In addition, the relative delay between the two pulse trains can be modified by a tunable delay line (TDL). The two pulse trains are then joined together and directed to the negative port of a balanced, differential detector. Since the difference between the central frequencies of the two replicas is outside the detector bandwidth, beating between the two is largely avoided. A reference pulse train is propagated in the lower branch of the setup, and detected at the positive port of the balanced detector. The relative delay and magnitude of the reference pulse train are controlled by a second EDFA and TDL. The electrical waveform at the balanced detector output can be expressed as:

$$
V(t) \propto\left|E_{\mathrm{in}}(t)\right|^{2}-a_{+}\left|E_{+}\left(t-t_{+}\right)\right|^{2}-a_{-}\left|E_{-}\left(t-t_{-}\right)\right|^{2}
$$
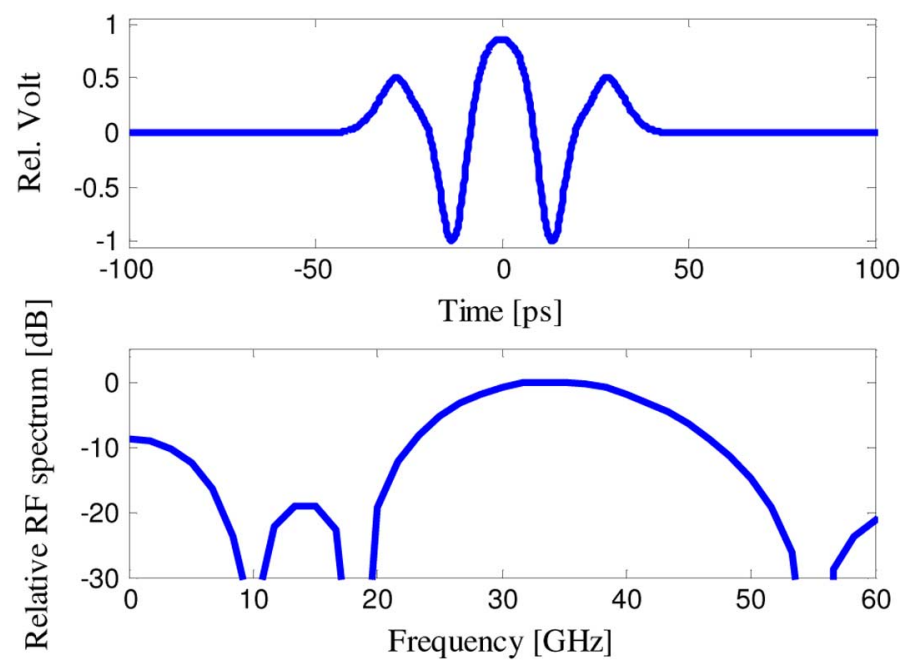

Fig. 4. Top-Simulated output waveform $V(t)$. The input pulse, HNLF and BPFs parameters are the same as those of Figs. 1 and 2, $a_{ \pm}=1.85$ and $t_{ \pm}=$ \pm 10 ps. Bottom-the corresponding RF spectrum $|\tilde{V}(\Omega)|^{2}$.

where $a_{ \pm}$and $t_{ \pm}$are the relative power levels and delays of the leading and trailing edge waveforms, respectively. Unless corrected by the TDLs, the relative delays $t_{ \pm}$correspond to $\max (\mathrm{min})$ of the input intensity derivative. The complete waveform design requires a numeric calculation. Nonetheless, the following relations may serve as useful starting points: $t_{+}=$ $-_{-} \approx(1 / 2) \tau_{0}, a_{ \pm} \approx(1 / 2) \int\left|E_{\text {in }}(t)\right|^{2} d t / \int\left|E_{+}(t)\right|^{2} d t$. The central frequency $f_{C}$ of $V(t)$ is on the order of $1 /\left|t_{2}-t_{1}\right|$, with $t_{1,2}$ defined in the previous section.

Fig. 4 shows a simulated example of the normalized shape of $V(t)$ (top panel) and its corresponding power spectral density $|\tilde{V}(\Omega)|^{2}$ (bottom), where $\Omega$ represents a radio frequency (RF) variable. The calculation parameters were the same as those of the previous section, with $a_{ \pm}=1.85$ and $t_{ \pm}= \pm 10 \mathrm{ps}$. The central frequency $f_{C}$ of the high-order, UWB output waveform is $34 \mathrm{GHz}$. The high and low $-10 \mathrm{~dB}$ cutoff frequencies $f_{H, L}$ are $47 \mathrm{GHz}$ and $23 \mathrm{GHz}$, respectively, providing a fractional bandwidth $B_{\mathrm{fr}} \equiv\left(f_{H}-f_{L}\right) / f_{C}$ of $70 \% . V(t)$ can be simply modified through changing the peak power, width and shape of the incoming pulse train, the detuning of the BPFs, and the relative magnitude and delay of the replica trains of narrowed pulses. Experimental generation of UWB waveform is described next.

\section{EXPERIMENT}

The generation of UWB pulses was demonstrated experimentally, using the setup of Fig. 3. The parameters of the input pulse train and the HNLF, as well as the settings of the BPFs, VOAs and TDLs were the same as those of the previous sections. The separation $T_{0}$ between neighboring pulses was $600 \mathrm{ps}$, corresponding to a data rate of $1.67 \mathrm{~Gb} / \mathrm{s}$. The average power of the amplified, input pulse train was $160 \mathrm{~mW}$. Fig. 5 shows the measured optical spectra $\left|\tilde{E}_{\mathrm{in}}(\lambda)\right|^{2},\left|\tilde{E}_{\mathrm{HNLF}}(\lambda)\right|^{2}$ and $\left|\tilde{E}_{ \pm}(\lambda)\right|^{2}$, corresponding to $E_{\mathrm{in}}(t), E_{\mathrm{HNLF}}(t)$ and $E_{ \pm}(t)$ respectively, as a function of wavelength $\lambda$. The imbalance between the two spectral sidelobes in $\left|\tilde{E}_{\mathrm{HNLF}}(\lambda)\right|^{2}$ is most likely due to residual dispersion in the HNLF. 


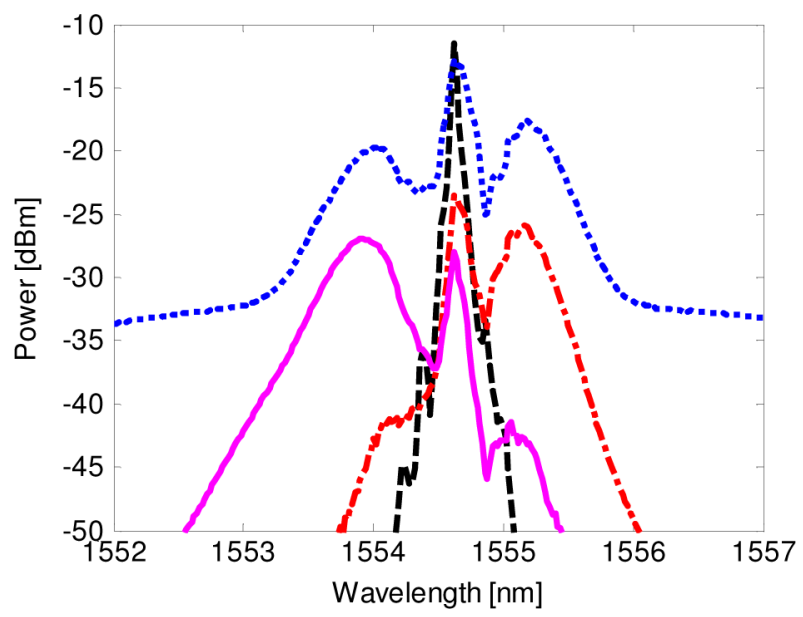

Fig. 5. Measured optical power spectra corresponding to $E_{\text {in }}(t)$ (dashed, black), $E_{\mathrm{HNLF}}(t)$ (dotted, blue), $E_{+}(t)$ (solid, magenta) and $E_{-}(t)$ (dashed-dotted, red). The experimental parameters were the same as those of the simulations in Fig. 4.


Fig. 6. Top-Simulated (solid, blue) and measured (dashed, red) output waveform $V(t)$. The experimental parameters were the same as those of the simulations in Fig. 4. Bottom - the corresponding simulated (solid, blue) and calculated experimental (dashed, red) RF spectra $|\tilde{V}(\Omega)|^{2}$.

Fig. 6 (top) shows the measured $V(t)$ alongside the corresponding simulation. The experimental RF spectrum $|\tilde{V}(\Omega)|^{2}$, calculated by taking the Fourier transform of the measured $V(t)$, is shown at the bottom of Fig. 6 alongside the simulated results. The experimental waveform generally agrees with the simulation. The difference between the spectral sidelobes in simulation and experiment can be accounted for by ps-scale errors in the relative delay of different paths, or an error of a few percent in the relative magnitude of different pulse trains. Such inaccuracies could also be responsible for the small-scale asymmetry in $V(t)$. Another possible source for asymmetry is residual dispersion in the HNLF (see also Fig. 5).

The flexibility of the waveform generation method is illustrated in Fig. 7 [20], [21], in which the setup parameters were adjusted to approximate the FCC mask for unlicensed indoor wireless UWB communication [2]. In this experiment, Gaussian pulses $(m=2)$ of width $\tau_{0}=100 \mathrm{ps}$, peak power $P_{\text {in }}=1 \mathrm{~W}$
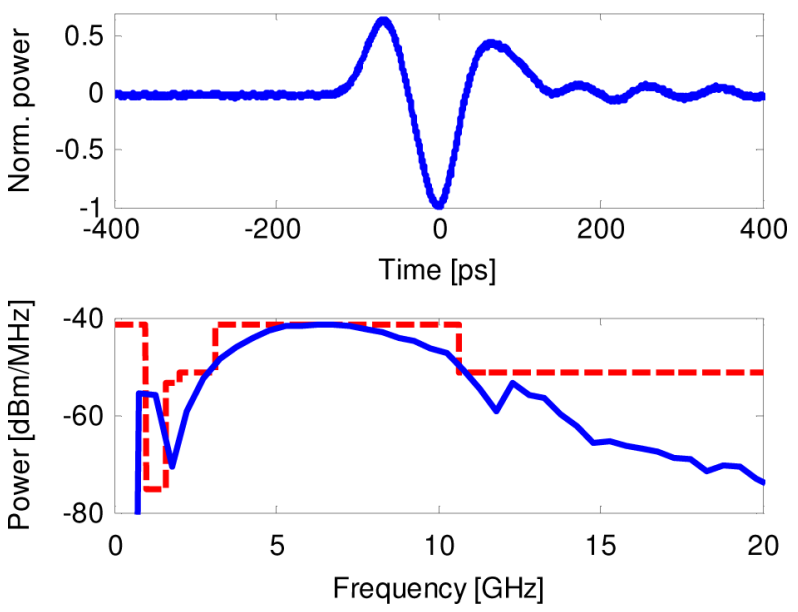

Fig. 7. Top-measured $V(t)$. Bottom-calculated experimental RF power spectrum $|\tilde{V}(\Omega)|^{2}$ (solid), alongside the FCC mask for indoor UWB communication [2] (dashed). Input pulses parameters: $P_{\text {in }}=1 \mathrm{~W}, \tau_{0}=100 \mathrm{ps,}$ $m=2, T_{0}=800 \mathrm{ps}$. HNLF parameters: $L=1 \mathrm{~km}, \gamma=11.3[\mathrm{~W} \cdot \mathrm{km}]^{-1}$. A single $40-\mathrm{GHz}$ wide BPF was detuned from $\omega_{0}$ by $\Delta \omega / 2 \pi=30 \mathrm{GHz}$.

and spacing $T_{0}=800 \mathrm{ps}$ were used. Only a single edge detection BPF was used in the experiment, with a spectral width of 40 $\mathrm{GHz}$ and detuning $\Delta \omega / 2 \pi$ of $30 \mathrm{GHz}$. The normalized output waveform $V(t)$ approximates a Gaussian doublet pulse shape (top panel). The calculated $|\tilde{V}(\Omega)|^{2}$ is drawn on the lower panel, alongside the FCC mask. The measurement generally complies with the mask requirements, although infringements can be seen at the lower frequency range. These infringements can be considerably reduced with the use of a narrower BPF, which was unavailable to us at the time of the experiment (see following discussion).

\section{DISCUSSION}

A technique for the photonic generation of UWB waveforms was demonstrated, based on all-optical detection of intensity edges of incoming super-Gaussian pulses. The shaping of the pulses takes place as they propagate in a UWB radio over fiber network architecture. To the best of our knowledge, previous demonstrations of photonic generation of high-order UWB waveforms required either mode-locked lasers or multiple sources. Numerous degrees of freedom are available for agile reconfiguration of the pulse shape. The technique could be particularly suitable for high-frequency waveforms, such as those intended for high-resolution vehicular radar systems. Waveforms that approximate the FCC spectral mask for indoor wireless communications were demonstrated as well [20], [21]. Better compliance with the FCC standard can be achieved with a choice of a narrower BPF: Fig. 8 shows an example of simulated $V(t)$ and $|\tilde{V}(\Omega)|^{2}$ obtained with a single $10-\mathrm{GHz}$ wide BPF. Results may be further improved by using two BPFs, as in Fig. 6.

The edge detectors were implemented based on SPM in a section of HNLF, and using two BPFs in parallel. However, both edges might be detected simultaneously with the application of just one band-stop optical filter centered at $\omega_{0}$, which would remove the center of the pulse (see Fig. 1). Data can be transmitted through simple on-off keying of the input pulses. On the other 

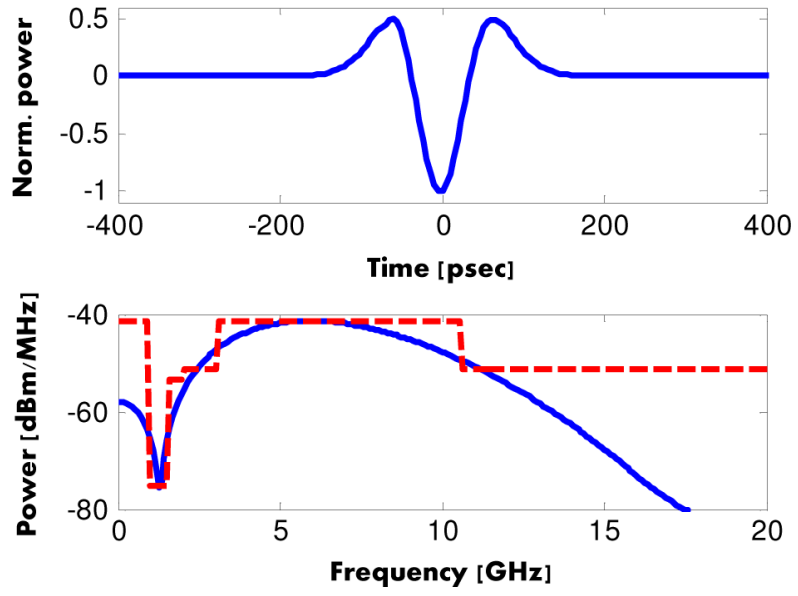

Fig. 8. Top-simulated $V(t)$. Bottom-corresponding simulated RF power spectrum $|V(\Omega)|^{2}$ (solid), alongside the FCC mask for indoor UWB communication [2] (dashed). Input pulses parameters: $P_{\text {in }}=1 \mathrm{~W}, \tau_{0}=70 \mathrm{ps}, m=2$. HNLF parameters: $L=1 \mathrm{~km}, \gamma=11.3[\mathrm{~W} \cdot \mathrm{km}]^{-1}$. A single $10-\mathrm{GHz}$ wide BPF was detuned from $\omega_{0}$ by $\Delta \omega / 2 \pi=20 \mathrm{GHz}$.

hand, pulse polarity modulation is not simply supported by the proposed approach.

The waveform generation setup includes multiple optical paths, the lengths of which were not matched in the experiment. The integrity of the UWB shape in a data-carrying, operational system could require path length equalization on $\mathrm{mm}$ scale (or a $1^{\circ} \mathrm{C}$ difference in temperature for the $1 \mathrm{~km}$-long fibers used above). The problem might be alleviated by using short fiber spans and high peak power levels, environmental isolation of fiber sections or active compensation. Alternatively, the relative delays $t_{ \pm}$could be controlled using dispersion rather than TDLs along different paths. The stability of the experimental setup was thus far validated over a couple of hours. Long term stability was not tested. The transmission of actual data using the proposed approach is the subject of further work.

The shaping and distribution scheme of the UWB pulses requires a two-fiber connection between a transmitter and a remote antenna element. Single-fiber transmission would be possible if a reference pulse shape $\left|E_{\text {in }}(t)\right|^{2}$ could be extracted from the SPM-broadened $E_{\mathrm{HNLF}}(t)$ at the receiver. Ideally $\left|E_{\mathrm{HNLF}}(t)\right|^{2}=\left|E_{\text {in }}(t)\right|^{2}$, however residual dispersion and EDFA noise might distort the reference pulse shape. A potential solution might be narrowband optical filtering centered at $\omega_{0}$. This modification will be examined in a further study.

The technique relies on off-the-shelf components only. Few of the components included in the experimental setup, such as EDFA, HNLF or differential detector, could be prohibitively expansive for certain applications. Higher cost may be more tolerable in applications in which a single transmitter is broadcasting to a large number of simple receivers, or where waveforms of high-order and high-frequency are required. Note that the transmission of $500 \mathrm{Mb} / \mathrm{s}$ data using the UWB pulses of Fig. 7, for example, requires an average power below $100 \mathrm{~mW}$, so that the EDFA could be rather modest. The HNLF can be replaced with a longer section of a more common fiber type. Cost-related aspects of the pulse generation method are addressed further in on-going work.
The comparison of the technique proposed in this work to previous approaches draws interesting analogies. Here, SPM introduces a time-to-frequency mapping, in which different temporal sections of the input pulses acquire different frequency shifts. This process is somewhat analogous to frequency-to-time mapping-based techniques [11]-[13], in which dispersion is used to assign a different delay to different spectral components of an input waveform. The subtraction of the intensity profile of delayed replicas from the original pulse shape might be viewed as a tapped-delay line filtering method. It should be noted, though, that the subtracted waveforms are obtained through nonlinear processing and are not scaled copies of the input. The nonlinear propagation enables the generation of higher-order waveforms while using only two replicas, and also allows for simple reconfiguration through input power adjustments. In conclusion, the approach demonstrated in this work might render the generation of complex, reconfigurable high-frequency UWB waveforms more accessible.

\section{REFERENCES}

[1] L. Yang and G. B. Giannakis, "Ultra-wideband communications: An idea whose time has come," IEEE Signal Process. Mag., vol. 21, no. 6, pp. 26-54, Nov. 2004.

[2] Federal Communication Commission, Tech. Rep. ET-Docket 98-153, FCC02-48, Apr. 2002

[3] J. Yao, F. Zeng, and Q. Wang, "Photonic generation of ultrawideband signals," J. Lightw. Technol., vol. 25, no. 11, pp. 3219-3235, Nov. 2007.

[4] F. Zeng and J. P. Yao, "All-optical bandpass microwave filter based on an electro-optic phase modulator," Opt. Exp., vol. 12, no. 16, pp. 3814-3819, 2004.

[5] J. Wang, F. Zeng, and J. P. Yao, "All-optical microwave bandpass filters implemented in a radio-over-fiber link," IEEE Photon. Technol. Lett., vol. 17, no. 8, pp. 1737-1739, 2005.

[6] Q. Wang, F. Zeng, S. Blais, and J. Yao, "Optical ultrawideband monocycle pulse generation based on cross-gain modulation in a semiconductor optical amplifier," Opt. Lett., vol. 31, no. 21, pp. 3083-3085, Nov. 2006

[7] H. Chen, M. Chen, T. Wang, M. Li, and S. Xie, "Methods for ultrawideband pulse generation based on optical cross-polarization modulation," J. Lightw. Technol., vol. 26, no. 15, pp. 2492-2499, Aug. 2008.

[8] M. Bolea, J. Mora, B. Ortega, and J. Capmany, "Optical UWB pulse generator using an $\mathrm{N}$ tap microwave photonic filter and phase inversion adaptable to different pulse modulation formats," Opt. Exp., vol. 17, no. 7, pp. 5021-5032, Mar. 2009.

[9] X. Yu, T. B. Gibbon, M. Pawlik, S. Blaaberg, and I. T. Monroy, "A photonic ultra-wideband pulse generator based on relaxation oscillations of a semiconductor laser," Opt. Exp., vol. 17, no. 12, pp. 9680-9687, Jun. 2009.

[10] X. Yu, T. B. Gibbon, and I. T. Monroy, "Experimental demonstration of all-optical 781.25 Mb/s binary phase-coded UWB signal generation and transmission," IEEE Photon. Technol. Lett., vol. 21, no. 17, pp. 1235-1237, Sep. 2009.

[11] C. Wang, F. Zeng, and J. Yao, "All-fiber ultrawideband pulse generation based on spectral shaping and dispersion-induced frequency-to-time conversion," IEEE Photon. Technol. Lett., vol. 19, no. 3, pp. 137-139, Feb. 2007.

[12] J. D. McKinney, I. S. Lin, and A. M. Weiner, "Shaping the power spectrum of ultra-wideband radio-frequency signals," IEEE Trans. Microw. Theory Tech., vol. 54, no. 12, pt. 1, pp. 4247-4255, Dec. 2006.

[13] M. Abtahi, M. Mirshafiei, J. Magne, L. A. Rusch, and S. LaRochelle, "Ultra-wideband waveform generator based on optical pulse-shaping and FBG tuning," IEEE Photon. Technol. Lett., vol. 20, no. 2, pp. 135-137, Jan. 2008.

[14] P. V. Mamyshev, "All optical data regeneration based on self-phase modulation effect," in Proc. ECOC, Madrid, Spain, Sep. 1998, pp. $475-476$.

[15] L. Thevenaz, "Slow and fast light in optical fibers," Nature Photonics, vol. 2, pp. 474-481, 2008 
[16] A. Loayssa, J. Capmany, M. Sagues, and J. Mora, "Demonstration of incoherent microwave photonic filters with all-optical complex coefficients," IEEE Photon. Technol. Lett., vol. 18, pp. 1744-1746, 2006.

[17] A. Zadok, A. Eyal, and M. Tur, "GHz-wide optically reconfigurable filters using stimulated Brillouin scattering," J. Lightw. Technol., vol. 25, pp. 2168-2174, Aug. 2007.

[18] J. Li, B. P.-P. Kuo, and K. K.-Y. Wong, "Ultra-wideband pulse generation based on cross-gain modulation in fiber optical parametric amplifier," IEEE Photon. Technol. Lett., vol. 21, no. 4, pp. 212-214, Feb. 2009.

[19] P. Velanas, A. Bogris, A. Argyris, and D. Syvridis, "High-speed all-optical first- and second-order differentiators based on cross-phase modulation in fibers," J. Lightw. Technol., vol. 26, no. 18, pp. 3269-3276, Sep. 2008.

[20] A. Zadok, X. Wu, J. Sendowski, A. Yariv, and A. E. Willner, in Frontiers in Optics (FiO): OSA Annual Meeting, San-Jose, CA, Oct. 2009, Paper FWK3.

[21] A. Zadok, X. Wu, J. Sendowski, A. Yariv, and A. E. Willner, "Photonic generation of ultra-wideband signals via pulse compression in a highly nonlinear fiber," IEEE Photon. Technol. Lett., vol. 22, no. 4, pp. 239-241, Feb. 2010.

[22] J. Xu, X. Zhang, J. Dong, D. Liu, and D. Huang, “All-optical differentiator based on cross-gain modulation in semiconductor optical amplifier," Opt. Lett., vol. 32, no. 20, pp. 3029-3031, 2007.

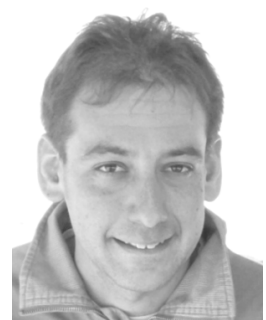

Avi Zadok received the B.Sc. degree (cum laude) in physics and mathematics from the Hebrew University of Jerusalem, Israel in 1994, the M.Sc. (cum laude) and the Ph.D. degrees (with distinction) in electrical engineering from Tel-Aviv University, Israel, in 1999 and 2007, respectively.

In 2007-2009 he was a Postdoctoral Research Fellow with the group of Prof. Amnon Yariv at the Department of Applied Physics at the California Institute of Technology, Pasadena CA. Since 2009, he has been an Assistant Professor at the School of Engineering of Bar-Ilan University, Ramat-Gan, Israel. He is the author of a book on dynamic optical filters, and the author and coauthor of 40 papers in international journals and conferences. His research interests include silicon photonics, optical fiber communication and signal processing, nonlinear optics and slow light phenomena.

Dr. Zadok is a member of the Optical Society of America (OSA). He was the recipient of the Clore Foundation fellowship for Ph.D. studies, and the Rothschild fellowship for post-doctoral studies.

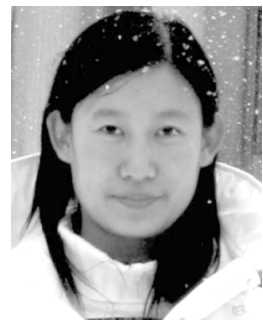

Xiaoxia Wu (S'06) received the B.E. degree from Jilin University, Changchun, China, in 2004, and the M.E. degree from Beijing University of Posts and Telecommunications (BUPT), Beijing, China, in 2006. She is currently working towards the Ph.D. degree in electrical engineering at University of Southern California, Los Angeles.

She was a visiting student at Consorzio Nazionale Interuniversitario per le Telecomunicazioni (CNIT), Pisa, Italy, in September 2008. She is the author or coauthor of more than 80 papers in prestigious international journals and conferences. Her current research interests include highspeed optical signal processing, optical performance monitoring and advanced optical modulation formats.

$\mathrm{Ms}$. Wu is a student member of the IEEE Photonics Society, the Optical Society of America and the International Society for Optical Engineering. She was one of the recipients of 2009 IEEE Photonics Society Graduate Student Fellowship Award. She is also a reviewer for IEEE PHOTONICS TECHNOLOGY LETTERs, IEEE PHOTONICS JouRnAL, Optics Letters, Optics Express and Optical Engineering.

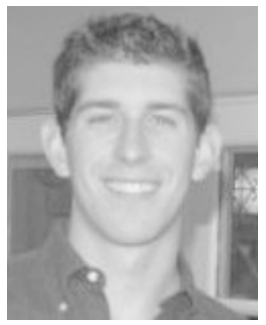

Jacob Sendowski (S'10) received the B.S. degree in electrical engineering from the University of California San Diego, in 2007 and the M.S. degree in electrical engineering from the California Institute of Technology, Pasadena, in 2009 where he is currently working toward the Ph.D. degree in electrical engineering.

His current research interests include semiconductor lasers, optical phase-lock loops, RF photonics, optoelectronics, OCT and non-linear optics.

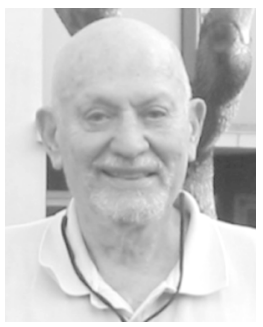

Amnon Yariv received the B.S., M.S. and Ph.D. degrees in electrical engineering from the University of California, Berkeley, in 1954, 1956, and 1958, respectively.

In 1959, he joined the Bell Telephone Laboratories, Murray Hill, NJ. In 1964, he joined the California Institute of Technology, Pasadena, as an Associate Professor of electrical engineering, where he became a Professor in 1966. In 1980, he became the Thomas G. Myers Professor of electrical engineering and applied physics. In 1996, he became the Martin and Eileen Summerfield Professor of applied physics and Professor of electrical engineering. On the technical and scientific sides, he took part (with various coworkers) in the discovery of a number of early solid-state laser systems, the original formulation of the theory of non-linear quantum optics, proposing and explaining mode-locked ultrashort-pulse lasers, GaAs optoelectronics; in proposing and demonstrating semiconductor based integrated optics technology, pioneering the field of phase conjugate optics, and proposing and demonstrating the semiconductor DFB laser. He has authored or coauthored widely in the laser and optics fields and has written a number of basic texts in quantum electronics, optics and quantum mechanics.

Dr. Yariv is a member of the American Academy of Arts and Sciences, the National Academy of Engineering, and the National Academy of Sciences.

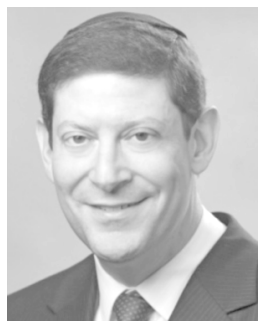

Alan E. Willner (S'87-M'88-SM'93-F'04) received the Ph.D. degree in electrical engineering from Columbia University, New York, in 1988.

He has worked at AT\&T Bell Laboratories and Bellcore. He is currently a Professor at the University of Southern California. He has 775 publications, including 2 books and 25 patents.

Prof. Willner is a Fellow of the Optical Society of America (OSA) and was a Fellow of the Semiconductor Research Corporation. His professional activities have included the following: President of the IEEE Lasers and Electro-Optics Society (LEOS), Editor-in-Chief of the IEEE/OSA JOURNAL OF LIGHTWAVE TECHNOLOGY, Editor-in-Chief of OSA Optics Letters, Editor-in-Chief of the IEEE JOURNAL OF SELECTED TOPICS IN QUANTUM ELECTRONICS, Co-Chair of the OSA Science and Engineering Council, General Co-Chair of the Conference on Lasers and Electro-Optics (CLEO), Chair of the IEEE TAB Ethics and Conflict Resolution Committee, General Chair of the LEOS Annual Meeting Program, Program Co-Chair of the OSA Annual Meeting, and Steering and Program Committee Member of the Conference on Optical Fiber Communications (OFC). He has received the National Science Foundation (NSF) Presidential Faculty Fellows Award from the White House, the Packard Foundation Fellowship, the NSF National Young Investigator Award, the Fulbright Foundation Senior Scholars Award, the IEEE LEOS Distinguished Traveling Lecturer Award, the USC University-Wide Award for Excellence in Teaching, and the Eddy Award from Pennwell for the Best Contributed Technical Article. 